\title{
The Impact of the Covid-19 Pandemic in Boccia Players with Cerebral Palsy in Brazil, Applying the Sf-36 Health Survey Questionnaire
}

\author{
Mirna Clemente $^{1^{*}}$, Marilis Dallarmi Miguel ${ }^{2}$, Darlan França Ciesielki Junior ${ }^{2}$, Ruth Eugenia \\ Cidade $^{2}$, Obdulio Gomes Miguel ${ }^{1}$ \\ ${ }^{1}$ Federal University of Parana (UFPR), Brazil \\ ${ }^{2}$ Physical Disability Association of Parana (ADFP), Brazil
}

*Corresponding Author: Mirna Clemente, Federal University of Parana (UFPR), Brazil

\begin{abstract}
:
Aim:This present study aimed to investigate if the Boccia players with cerebral palsy during the COVID-19 quarantine period demonstrated traces of depression, anxiety, or any other emotional issues when their Boccia training routine was compared.
\end{abstract}

Methods: Twelve people with cerebral palsy were asked to complete the informed consent form and the questionnaire. They were from the Physical Disability Association of Parana (ADFP) and Boccia players. All of them, after inclusion and exclusion criteria, were invited to answer the 36-Item Short-Form Health Survey (SF-36) in May 2019 during their training period and in June 2020 when Brazil was the epicenter of the Pandemic, and they were in quarantine with Boccia training restriction and social distancing for about hundred days.

Results: The data demonstrated a statically significant difference, with lower levels when the training period was compared to the COVID-19 quarantine period on people with cerebral palsy, on Role Physical Limitation, General health, Vitality, Social Functioning, Role Emotional Limitation, Mental health, the Physical Composite Score and the Mental Composite Score. In contrast, the physical function and bodily pain were not statically different, showing no impact in these two physical scores

Conclusion: In light of the aforementioned, the COVID-19 quarantine period with social distancing and no sport participating affected negatively psychological and some physical parameters om people with cerebral palsy. Further studies are necessary to assess the impact of COVID-19 on other populations such as Down syndrome, in order to find ways to protect these vulnerable people's mental health during the pandemic

Keywords: Covid-19, Boccia players, cerebral palsy, SF-36, and mental health

\section{INTRODUCTION}

The Coronavirus disease 2019 (COVID-19), which has been spreading very fast all over the world in 2020, started in early December 2019 in ChinaGagnier et al. (2013)Guan et al. (2020). Hence, on March 11, 2020, the World Health Organization (WHO) declared COVID-19 as a global pandemic Cucinotta; Vanelli (2020). The COVID-19 pandemic has demonstrated to affect the political, medical, social, and economic system, so far Jakovljevic et al. (2020).Besides, people with diabetes, hypertension, cardiovascular diseases, and respiratory problems have been demonstrated to be more vulnerable to acquire the severe COVID-19 form Lai et al. (2020).

Regarding cerebral palsy, this condition has been associated with speech disturbance, epilepsy, nutritional problems, motor coordination, spasticity, costructures, fatigue, pain, lung disease and intellectual impairments Zolezzi et al. (2017). There are shreds of evidence that around $25 \%$ of adults with cerebral palsy have demonstrated depression symptoms clinically analyzed Smith et al. (2019)Van der Slot et al. (2012). It is believed that Paralympic sport or Para sport, practiced by people with cerebral palsy, could be a very good strategy to improve their health-related to quality of life and promote psychological well-being Clemente et al. (2019)Blauwet (2005) Yazicioglu et al. (2012)Shapiro; Malone (2016) 
Cerebral palsy is not considered a risk factor from COVID-19, nonetheless, some specific comorbidities in these vulnerable people could be a problemMcPhee et al. (2019)Ben-Pazi et al. (2020). Moreover, the psychological, economic, and social of the COVID-19 pandemic on people with cerebral palsy have been demonstrated extensively Ben-Pazi et al. (2020).

This study applied the Medical Outcomes Study 36-Item Short-Form Survey (SF-36) to measure the Health-Related to Quality of Life (HRQL) by physical functioning, role limitation due to physical health problems, bodily pain, general health perceptions, vitality, social functioning, role limitation due to emotional problems and mental health Campolina, AG; Bortoluzzo, AB; Ferraz, MB; Ciconelli (2010).

Since COVID-19 pandemic has been associated with mental health consequences, mostly because of interventions such as social distancing, isolation,lockdown or quarantineTorales et al. (2020). The present study aimed to investigate if the Boccia players with cerebralpalsy during theCOVID-19 quarantine period demonstrated traces of depression, anxiety, any other emotional issues or psychological impairment, when their Boccia training routine was compared.

\section{MATERiAl AND MethodS}

\subsection{Study Design and Ethics Approval}

This is acase study. The local ethics committee (Federal University of Paraná- Brazil) under the number 2.294.303. The protocol for carrying out the study was developed, by following the protocol of Consensus-based Clinical Case Reporting Guideline Development (CARE) guidelines Gagnier et al. (2013).

\subsection{Demographic Characteristics Questions}

Specific questions about age, education, family income, and functional classification in the Paralympic sport were asked. Cerebral palsy people who start to participate in Boccia competition must be classified according to Boccia International Sport Federation rules. Boccia classification (BC) is divided:

1. ClassBC1 has severe activity limitations affecting their legs, arms, and trunk control due to coordination impairments. These athletes can grasp and throw the ball with no assistive devices. This class allows people with $\mathrm{CP}$ with some leg control, assisting them in using their feet to play with a ball.

2. Class $\mathrm{BC} 2$ have better trunk control and arm function than the players in classes $\mathrm{BC} 1$ and $\mathrm{BC} 3$. The abilities of their arms and hands often allow them to throw the ball overhand and underhand as well as with a variety of grasps

3. Class BC3 has significantly limited functions in their arms and legs, along with poor or no trunk control. To help them propel the ball onto the court, they use a ramp and other assistive devices to roll the ball.

4. Class BC4 comprises of athletes with impairments that have no cerebral origin. Muscular dystrophy, spinal cord injuries, and amputations affecting all four limbs are among the possible health conditions BISFed (2018)

\subsection{Participants and Procedures}

Twelve people with cerebral palsy were invited to complete the informed consent form before the questionnaire. These people are from the Physical Disability Association of Parana (ADFP) andwere asked to answer the 36-Item Short-Form Health Survey (SF-36) in May 2019 (by the internet) during their training period and in June 202, when Brazil was the epicenter of the Pandemic, and they were in quarantine with no Boccia training and with social restrictions for about hundred days.

$>$ Inclusion criteria

1. 18 years old and older

2. Be associated with the ADFP

3. Practicing Boccia for over two years 
4. Be diagnosed with cerebral palsy.

$>$ Exclusion criteria

1. Using anxiolytic or depression medicines

2. Being diagnosed with depression or any other mental disease

\subsection{Questionnaire}

This study used the SF-36 to measure the quality of life people with cerebral palsy, validated in Brazil Campolina, AG; Bortoluzzo, AB; Ferraz, MB; Ciconelli (2010)

The SF-36 is composed of 36 questions, which are used to calculate eight scales of different health dimensions:

1. Physical Functioning (PF); estimate the limitations related to physical capacity;

2. Role Physical Limitation (RPL); evaluate the constraints of the type of work and connects the tasks of practical daily life;

3. Bodily Pain (BP): assesses the existence of pain, its intensity, and how much it limits day-to-day activities;

4. General health status $(\mathrm{GH})$; check the general health of the patient out;

5. Vitality (V); analyze vigor, energy, and fatigue;

6. Social Functioning (SF); estimate socialization;

7. Role Emotional Limitation (REL): assesses aspects of the psychological well-being of the patient;

8. Mental health $(\mathrm{MH})$ : evaluate the patient's anxiety, depression, behavior, and psychological wellbeing.

The first four scales can create the Physical Composite Score (PCS), while the last four create the Mental Composite Score (MCS), which provides a measure of the overall effect of physical and psychological impairment. Scores for the SF-36 scales range between 0 and 100, with higher scores indicating better quality of life. This questionnaire has been recommended and trusted, and it was used during rehabilitation procedures with a definitive diagnosis of COVID-19 Sonja A. Rasmussen, MD, MS (2020)

\subsection{Statistics}

GraphPad Prism Software (GraphPad Software, Inc. La Jolla, CA, USA, version 7.0) was used and the significance was considered at a $p$-value $<0.05$. Statistical analysis was carried out applying the one-way analysis of variance (ANOVA) with Tukey's multiple comparison tests and/or KruskalWallis test, followed by Dunn's test if the requirements for performing a parametric test were not satisfied. Results were shown as mean \pm S.E.M.

\section{Results}

Twelve people were invited and selected to participate in this study, after following the inclusion and exclusion criteria. Then, they completed the informed consent form demographic characteristics questions and the SF-36 questionnaire twice, in June 2019 during the training period of Paralympic sport in this case, Boccia, and in July 2020 during the COVID-19 quarantine period in Curitiba, Parana, Brazil with sport and social restriction.

The demographic characteristic was described in table 1, and the result of age was described in $\%$ of 2020 ,.All of the participants were diagnosed with cerebral palsy since they were born.

Table1. Demographic characteristics of Boccia players with cerebral palsy

\begin{tabular}{|l|l|}
\hline Demographiccharacteristics & Numberor \% \\
\hline Age & $33.67 \%$ \\
\hline Gender & \\
Male & 8 \\
Female & 4 \\
\hline
\end{tabular}


The Impact of the Covid-19 Pandemic in Boccia Players with Cerebral Palsy in Brazil, Applying the Sf-36 Health Survey Questionnaire

\begin{tabular}{|l|l|}
\hline \multicolumn{2}{|l|}{} \\
\hline Family income & 0 \\
Good & 6 \\
Moderate & 6 \\
Poor & \\
\hline Education & 0 \\
No education & 5 \\
Lower secondary school & 7 \\
Secondaryschoolcompleted & \\
\hline Frequency of Boccia training & 2 \\
Once a week & 1 \\
Twice a week & 9 \\
Three times or more & \\
Boccia classification & 2 \\
BC1 & 6 \\
BC2 & 4 \\
BC3 & 0 \\
BC4 & \\
\hline
\end{tabular}

The characteristics of the boccia players with cerebral palsy assessed by SF-36 questionnaire and the results by physical PF, RPL, BP, GH, V, SF, REL, MH, PCS, MCS, standard error of the mean (S.E.M.), during the training period in 2019 and the COVID-19 quarantine period with training and social restriction. The data demonstrated a statically significant difference, with lower levels when the training period was compared to the COVID-19 quarantine period on people with cerebral palsy, on RPL, GH, V, SF, REL, MH, PCS, and MCS. In contrast, the PF and BP were not statically different, showing no impact in these two physical scores (Table 2, Figures 1 and 2).

Table2. Finding of SF-36 scales on Boccia players with cerebral palsy

\begin{tabular}{|l|lc|lc|}
\hline SF-36 SCALES & \multicolumn{3}{|l|}{$\begin{array}{l}\text { Boccia players } \\
\text { During the training period } \\
\text { N=12 } \\
\text { MEAN }\end{array}$} & $\begin{array}{l}\text { Boccia players } \\
\text { During COVID-19 quarantine period } \\
\text { N=12 }\end{array}$ \\
SE M & 4.24 & 17.50 & S.E.M \\
\hline RF & 17.92 & 12.87 & $40.83 *$ & 4.24 \\
\hline BP & 66.25 & 8.04 & 66.67 & 11.21 \\
\hline GH & 77.75 & 1.88 & $65.67 *$ & 3.18 \\
\hline V & 78.58 & 3.22 & $69.58^{*}$ & 4.62 \\
\hline SF & 82.50 & 4.09 & $67.67 *$ & 8.06 \\
\hline REL & 90.83 & 5.94 & $57.61 *$ & 8.55 \\
\hline MH & 90.62 & 4.27 & $70.00 *$ & 5.24 \\
\hline
\end{tabular}

* Statistically significant difference between groups $\mathrm{p}<0.05$

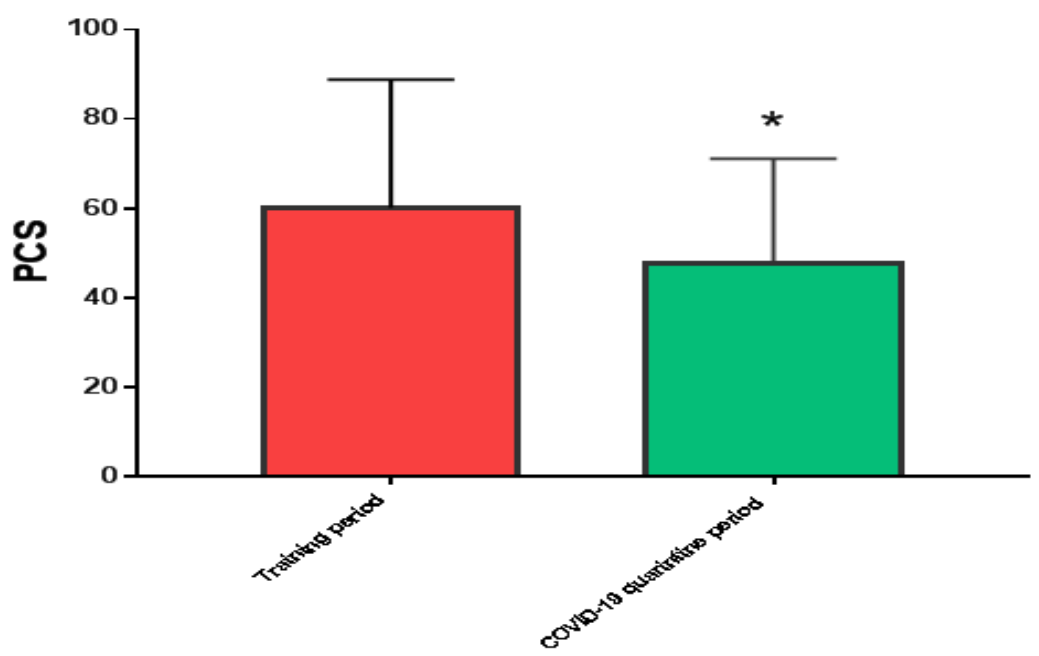

Figure1. Effect of a COVID-19 quarantine period with social and exercise restriction on people with cerebral palsy on PCS comparing to the training period*significantly different from pre-value, paired t-test, $p<0.05$ 


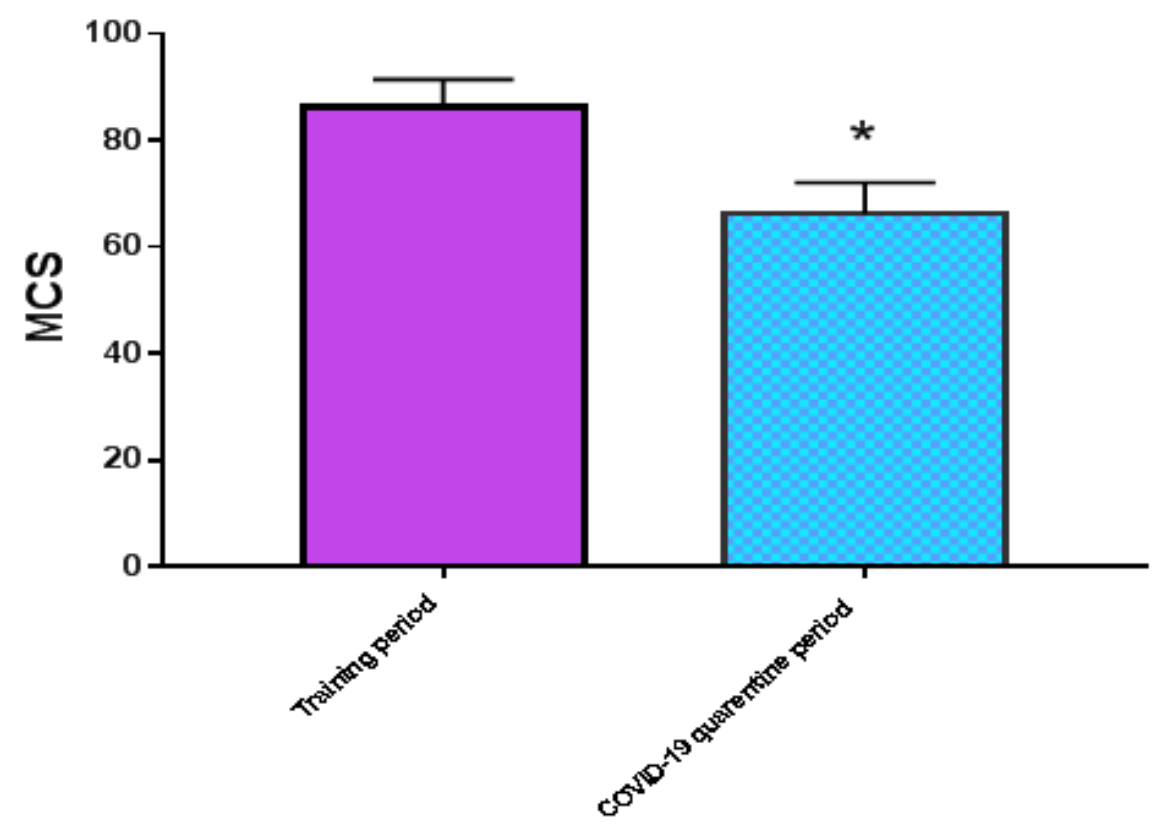

Figure2. Effect of a COVID-19 quarantine period with social and exercise restriction on people with cerebral palsy on MCS comparing to the training period*significantly different from pre-value, paired $t$-test, $p<0.05$

\section{DISCUSSION}

Researchers, on the psychological effects of quarantine during past pandemics such as Ebola and H1N1, showed psychological distress Mazza et al. (2020). The COVID-19 pandemic has been describing to affect not only physical health, but mental health and psychological well-being as well. People are exposed to a stressful situation such as home confinement, isolation, and social distancing, causing anxiety, sleep problems and depression Kontoangelos et al. (2020).

Therefore, the present research aimed to investigate if the Boccia players with cerebral palsy during the quarantine period of the COVID-19 pandemic demonstrated traces of depression, anxiety, or any other emotional issues comparedwith their Boccia training routine. Although there are studies involving SF-36 and Paralympic sport Clemente et al. (2019)“19-30_Clemente fencers.pdf” ()and SF36 against COVID-19 Sonja A. Rasmussen, MD, MS (2020), no one have shown the impact of this pandemic in athletes with cerebral palsy. We hypothesized that social life and physical exerciserestriction might affect negatively, in terms of psychological well-being, on people with cerebral palsy.

The first four scales which represent the physical domains (PF, RPL, BP, and GH) and create the PSC. The results demonstrated that RPL, GH, and PSC were the statistically significant different, with lower levels when the training period was compared to the COVID-19 quarantine period on people with cerebral palsy. In contrast, the PF and BP were no significant, showing no impact in these two physical scores. Since the sport has been used to promote health on people with disability and may enhance their functional independence Blauwet (2005)Blauwet; Willick (2012), the results which indicated physical function declining, could be justified.

It has been widely accepted around the world that sports participation can improve mental health, decreasing anxiety, insomnia, and depression Shapiro; Malone (2016) and provide opportunities for disabled people demonstrated by psychological functioning Yazicioglu et al. (2012). Besides. Fiorilli et al. (2013) showed in their study that Paralympic sport could stimulate vigor, vitality and alleviate tension, fatigue, and depression.

The last four scales represent physiological impairment (V, SF, REL, and MH), with these four is created MSC. The data of this study showed that all the physiological parameters (V, SF, REL, MH, and MSC) were statistically different. Thus, the evidence suggeststhat the COVID-19 pandemic had a negative impact on the mental health of people with cerebral palsy with sport restriction and social distancing, corroborating with Rajkumar (2020) in his narrative review, which associated COVID-19 
pandemic with mental health problems. Furthermore, the data is very consistent with other studies that linked COVID-19 and its impact on global mental health Torales et al. (2020).

All this finding support the big importance of conducting more studies with vulnerable people applying different questionnaires or methodologies to understand the impact of this pandemic on mental health and health-related to quality of life.

\section{LiMitation}

This study has demonstrated some limitations, which may affect the results negatively, the small size of the study sample and the first interview in 2019 was done presently and the second, during the COVID-19 pandemic in 2020, was done by the internet since they were going through the social distance.

\section{CONCLuSion}

In lightof the proceeding discussion, the COVID-19 quarantine period with social distancing and no sport participating affected negatively psychological and some physical parameters on people with cerebral palsy. Further studies are necessary to assess the impact of the COVID-19 on other populations such as Down syndrome, in order to find ways to protect these vulnerable people's metal health.

\section{ACKNOWLEDGMENTS}

We gratefully acknowledge Physical Disability Association of Parana for their assistance with this project.

\section{REFERENCES}

[1] 19-30_Clemente fencers.pdf. .

[2] BEN-PAZI, H.; BENI-ADANI, L.; LAMDAN, R. Accelerating Telemedicine for Cerebral Palsy During the COVID-19 Pandemic and Beyond. Frontiers in Neurology, v. 11, n. June, p. 1-7, 2020.

[3] BISFED. Boccia Classification Rules. Boccia International Sports Federation. Anais.... p.4th Edition, 2018.

[4] BLAUWET, C. Promoting the Health and Human Rights of Individuals with a Disability through the Paralympic Movement. , , n. January, 2005.

[5] BLAUWET, C.; WILLICK, S. E. The paralympic movement: Using sports to promote health, disability rights, and social integration for athletes with disabilities. PM and R, v. 4, n. 11, p. 851-856, 2012.

[6] CAMPOLINA, AG; BORTOLUZZO, AB; FERRAZ, MB; CICONELLI, R. Validação da versão brasileira do questionario genérico de qualidade de vida SF 6D. , v. 66, p. 3103-3110, 2010. Disponível em: 〈http://www. scielo.br/pdf/csc/v16n7/10.pdf >. .

[7] CLEMENTE, M.; MIGUEL, M. D.; FELIPE, K. B.; et al. Comparative Study of the Psychological WellBeing of Healthy Volunteers, Paralympic Athletes and Non-Athletes with Spinal Cord Injury, Assessed by Short Form Survey (SF-36). International Journal of Sports and Physical Education, v. 5, n. 2, p. 23-29, 2019.

[8] CUCINOTTA, D.; VANELLI, M. WHO declares COVID-19 a pandemic. Acta Biomedica, v. 91, n. 1, p. 157-160, 2020.

[9] FIORILLI, G.; IULIANO, E.; AQUINO, G.; et al. Mental health and social participation skills of wheelchair basketball players: A controlled study. Research in Developmental Disabilities, v. 34, n. 11, p. 3679-3685, 2013. Elsevier Ltd. Disponível em: 〈http://dx.doi.org/10.1016/j.ridd.2013.08.023>. .

[10] GAGNIER, J. J.; KIENLE, G.; ALTMAN, D. G.; et al. The CARE guidelines: Consensus-based clinical case reporting guideline development. Headache, v. 53, n. 10, p. 1541-1547, 2013.

[11] GUAN, W.; NI, Z.; HU, Y.; et al. Clinical characteristics of coronavirus disease 2019 in China. New England Journal of Medicine, v. 382, n. 18, p. 1708-1720, 2020.

[12] JAKOVLJEVIC, M.; BJEDOV, S.; JAKSIC, N.; JAKOVLJEVIC, I. Covid-19 pandemia and public and global mental health from the perspective of global health security. Psychiatria Danubina, v. 32, n. 1, p. 6$14,2020$.

[13] KONTOANGELOS, K.; ECONOMOU, M.; PAPAGEORGIOU, C. Mental Health Effects of COVID-19 Pandemia: A Review of Clinical and Psychological Traits. Psychiatry Investigation, v. 17, n. 6, p. 491$505,2020$. 
[14] LAI, A. L.; MILlET, J. K.; DANIEL, S.; FREED, J. H.; WHITTAKER, G. R. prevalence of comorbidities and its effects in patients infected with SARS-CoV-2. the Lancet, v. 395, n. April, p. 1315, 2020.

[15] MAZZA, C.; RICCI, E.; BIONDI, S.; et al. A nationwide survey of psychological distress among italian people during the covid-19 pandemic: Immediate psychological responses and associated factors. International Journal of Environmental Research and Public Health, v. 17, n. 9, p. 1-14, 2020.

[16] MCPHEE, P. G.; MACDONALD, M. J.; CHENG, J. L.; DUNFORD, E. C.; GORTER, J. W. Emerging evidence for accelerated ageing and cardiovascular disease in individuals with cerebral palsy. Journal of Rehabilitation Medicine, v. 51, n. 7, p. 525-531, 2019.

[17] RAJKUMAR, R. P. COVID-19 and mental health: A review of the existing literature. Asian Journal of Psychiatry, v. 52, p. 102066, 2020. Elsevier B.V. Disponível em: <https://doi.org/10.1016/j.ajp.2020.102066>. .

[18] SHAPIRO, D. R.; MALONE, L. A. Quality of life and psychological affect related to sport participation in children and youth athletes with physical disabilities: A parent and athlete perspective. Disability and Health Journal, v. 9, n. 3, p. 385-391, 2016. Elsevier Inc. Disponível em: <http://dx.doi.org/10.1016/j.dhjo.2015. 11.007>. .

[19] VAN DER SLOT, W. M. A.; NIEUWENHUIJSEN, C.; VAN DEN BERG-EMONS, R. J. G.; et al. Chronic pain, fatigue, and depressive symptoms in adults with spastic bilateral cerebral palsy. Developmental Medicine and Child Neurology, v. 54, n. 9, p. 836-842, 2012.

[20] SMITH, K. J.; PETERSON, M. D.; O’CONNELL, N. E.; et al. Risk of Depression and Anxiety in Adults with Cerebral Palsy. JAMA Neurology, v. 76, n. 3, p. 294-300, 2019.

[21] SONJA A. RASMUSSEN, MD, MS, J. C. S. Since January 2020 Elsevier has created a COVID-19 resource centre with free information in English and Mandarin on the novel coronavirus COVID-. Ann Oncol, , n. January, p. 19-21, 2020.

[22] TORAlES, J.; O’HIGGINS, M.; CASTALDELli-MAIA, J. M.; VENTRIGLIO, A. The outbreak of COVID-19 coronavirus and its impact on global mental health. International Journal of Social Psychiatry, v. 66, n. 4, p. 317-320, 2020.

[23] YAZICIOGLU, K.; YAVUZ, F.; GOKTEPE, A. S.; TAN, A. K. Influence of adapted sports on quality of life and life satisfaction in sport participants and non-sport participants with physical disabilities. Disability and Health Journal, v. 5, n. 4, p. 249-253, 2012. Elsevier Inc. Disponível em: <http://dx.doi.org/10.1016/j. dhjo.2012.05.003>.

[24] ZOLEZZI, M.; ABDULRHIM, S.; ISLEEM, N.; ZAHRAH, F.; ELTORKI, Y. Medical comorbidities in patients with serious mental illness: a retrospective study of mental health patients attending an outpatient clinic in Qatar. Neuropsychiatric disease and treatment, v. 13, p. 2411-2418, 2017.

Citation: Mirna Clemente, et.al. "The Impact of the Covid-19 Pandemic in Boccia Players with Cerebral Palsy in Brazil, Applying the Sf-36 Health Survey Questionnaire "International Journal of Sports and Physical Education (IJSPE), vol 7, no. 2, 2021, pp.01-07. doi: https://doi.org/10.20431/2454-6380.0702001.

Copyright: (c) 2021 Authors. This is an open-access article distributed under the terms of the Creative Commons Attribution License, which permits unrestricted use, distribution, and reproduction in any medium, provided the original author and source are credited. 